\title{
Blood Pressure is the Strongest Component Associated with Arterial Stiffness in Mexican Patients with Metabolic Syndrome
}

\author{
Ernesto G Cardona Muñoz, Fernando Grover Páez*, Carlos G Ramos Becerra, \\ Guillermo A Alanis Sánchez, Assen Ognianov Iantchoulev, David Cardona Müller, \\ Sylvia E Totsuka Sutto, Sara Pascoe González
}

Institute of Experimental and Clinical Therapeutics, Department of Physiology, University Center for Health Sciences, University of Guadalajara, MEXICO

*Corresponding Contact:

Email: fgroverp@ hotmail.com

Phone: +52110585200

\begin{abstract}
Objective: To establish which of the metabolic syndrome (MetS) components are more related to arterial stiffness. Research Design and Methods: We recruited eighty-two non-smoking male patients, 40 to 77 years old, (42 without MetS and 41 with MetS). The local ethics committee approved the study and signed informed consent forms were obtained from patients. Body mass index (BMI) was calculated as weight (in kilograms) divided by the square of height (in meters), and waist measurements were according to WHO recommendations. Blood pressure and baPWV were measured with the “Vascular Profiler 1000" device (VP-1000) (Omron®, Kyoto, Japan). All blood sample determinations were obtained after a 12-h fasting period and processed within one $\mathrm{h}$. We used an oxidase technique to measure fasting plasma glucose (FPG) and enzymatic methods to obtain total cholesterol, high-density lipoprotein cholesterol (HDL-c) and triglyceride values. Low-density lipoprotein cholesterol (LDL-c) was estimated using the Friedewald formula (LDL-C = Total Cholesterol - HDL-C - Triglycerides/5). Results: We observed strong and significant correlations between SBP and baPWV $(\mathrm{r}=.753, \mathrm{p}=<0.001)$, and between SBP and PP $(\mathrm{r}=.853, \mathrm{p}=<0.001)$. The remaining components did not correlate with baPWV or PP.
\end{abstract}

Keywords: Arterial stiffness, blood pressure, cardiovascular disease, metabolic syndrome, brachial-ankle pulse wave velocity, pulse, pressure

How to Cite: Muñoz EG, Páez* FG, Becerra CG, Sánchez GA, lantchoulev AO, Müller DC, Sutto SE and González SP. 2015. Blood Pressure is the Strongest Component Associated with Arterial Stiffness Inmexican Patients with Metabolic Syndrome ABC Journal of Advanced Research, 4, 129-132.

This article is is licensed under a Creative Commons Attribution-NonCommercial 4.0 International License.

Attribution-NonCommercial (CC BY-NC) license lets others remix, tweak, and build upon work non-commercially, and although the new works must also acknowledge \& be non-commercial.

\section{INTRODUCTION}

The prevalence of metabolic syndrome in Mexico increased from $26.6 \%$ to $45 \%$ in 2012 (Barquera, 2012). In the United States, the highest prevalence of metabolic syndrome was 
in Mexican Americans compared to other Latin American populations, placing this group at high cardiovascular risk (Allison, 2008).

The path physiology by which MetS increases cardiovascular risk is only partially understood, and the relationship between MetS and AS still remains unclear.

AS is an important independent predictor of cardiovascular morbidity and mortality (Laurent, 2001). Carotid-femoral pulse wave velocity (cfPWV) is considered the gold standard for AS evaluation (Laurent, 2006).

Brachial-ankle pulse wave velocity (baPWV) is a reliable approach to assess AS. Studies have shown a positive correlation between baPWV and cfPWV ( $r=0.73)$ (Tanaka, 2009). The association between components of MetS and AS in the Mexican population remains undetermined. This cross-sectional study aimed to evaluate the correlation between the components of MetS and AS.

\section{Materials and Methods}

Eighty-two non-smoking male patients, 40 to 77 years old, were included (42 without MetS and 41 with MetS). The study was performed in accordance with the ethical standards put forth in the 1964 Declaration of Helsinki and its later amendments. The local ethics committee approved the study and signed informed consent forms were obtained from patients. Study participants were instructed to abstain from drinking alcoholic and caffeinated beverages 12 hours prior to the evaluation. Individuals adopted a supine position and rested for 15 minutes before examination. One operator performed all the measurements. Body mass index (BMI) was calculated as weight (in kilograms) divided by the square of height (in meters), and waist measurements were according to $\mathrm{WHO}$ recommendations.

Blood pressure and baPWV were measured with the "Vascular Profiler 1000" device (VP1000), Omron ${ }^{\circledR}$, Kyoto, Japan). The methodological details have been described previously (Yamashina, 2002). Mean right and left baPWV values were used for the analysis. All blood sample determinations were obtained after a 12-h fasting period and processed within oneh. We used an oxidase technique to measure fasting plasma glucose (FPG) and enzymatic methods to obtain total cholesterol, high-density lipoprotein cholesterol (HDL-c) and triglyceride values. All of these measurements were performed using commercially available kits (Beckman Instruments Inc, Brea, Calif.) with intra-assay and inter-assay coefficients of variation less than 3\%. Low-density lipoprotein cholesterol (LDL-c) was estimated using the Friedewald formula (LDL-C $=$ Total Cholesterol - HDL-C - Triglycerides $/ 5$ ).

\section{Statistical ANALYSis}

Statistical analysis was conducted using SPSS version 20.0 (SPSS Inc., Chicago, IL, USA). All values are expressed as an average \pm the standard deviation (SD); the association between MetS and AS measurements was analyzed using Pearson's correlation coefficient. All p-values were two-tailed, and $\mathrm{P}<0.05$ was accepted as statistically significant.

\section{Results}

Table 1 shows a list of the patients' characteristics. The two groups were similar regarding mean age, BMI, and waist circumference. We observed significant differences in FPG, HDL-c, systolic blood pressure (SBP), diastolic blood pressure (DBP) and triglyceride levels, but with no statistically significant difference in LDL-c, or total cholesterol 
concentrations. Concerning AS parameters, we observed statistical differences in baPWV and peripheral pulse pressure (PP).

Table 1: Anthropometric, metabolic, hemodynamic, and arterial stiffness characteristics of patients without $(n=42)$ and with $(n=41)$ metabolic syndrome

\begin{tabular}{|l|l|l|l|}
\hline Data & No $(\mathrm{n}=42)$ & Yes $(\mathrm{n}=41)$ & $p^{a}$ \\
\hline Age, years & $55.2 \pm 7.8$ & $58.1 \pm 7.4$ & .125 \\
\hline BMI, kg/m ${ }^{2}$ & $25.0 \pm 3.2$ & $28.7 \pm 2.6$ & .002 \\
\hline Waist, $\mathrm{cm}$ & $80.5 \pm 9.1$ & $101.81 \pm 7.1$ & .008 \\
\hline FPG, mg/dL & $90 . \pm 12.1$ & $101.4 \pm 19.9$ & .018 \\
\hline Triglycerides, mg/dL & $118.6 \pm 62.9$ & $194.1 \pm 31.4$ & .002 \\
\hline HDL-c, mg/dL & $57.6 \pm 14.9$ & $48.5 \pm 14.4$ & .019 \\
\hline LDL-c, mg/dL & $98.5 \pm 34.7$ & $103.3 \pm 42.1$ & .653 \\
\hline Total cholesterol, mg/dL & $182.5 \pm 26.3$ & $175.1 \pm 47.6$ & .524 \\
\hline Systolic blood pressure, $\mathrm{mmHg}$ & $123.3 \pm 7.8$ & $146.4 \pm 18.0$ & $<0.01$ \\
\hline Diastolic blood pressure, $\mathrm{mmHg}$ & $76.1 \pm 7.2$ & $86.4 \pm 9.4$ & $<0.01$ \\
\hline Peripheral PP*, mmHg & $47.3 \pm 9.4$ & $59.7 \pm 15.4$ & $<0.01$ \\
\hline Mean baPWV ${ }^{* *}, \mathrm{~cm} / \mathrm{s}$ & $1347.0 \pm 173.5$ & $1578.7 \pm 295.0$ & $<0.05$ \\
\hline Metabolic Syndrome & \multicolumn{3}{|l}{} \\
\hline
\end{tabular}

aThe Two-tailed Student t-test was used to analyze the between-group difference of continuous variables; ${ }^{*}=\mathrm{PP} ;{ }^{* *}=$ brachial-ankle pulse wave velocity.

Regarding the association of AS and MetS components (Table 2), among patients with MetS, we found a statistically significant and strong correlation between SBP and baPWV $(\mathrm{r}=.753, \mathrm{p}=<0.001)$, and between SBP and $\mathrm{PP}(\mathrm{r}=.853, \mathrm{p}=<0.001)$. The remaining components did not correlate with baPWV or PP.

Table 2: Correlation between the different components of metabolic syndrome and arterial stiffness $(n=41)$

\begin{tabular}{|l|l|l|l|l|}
\hline Data & baPWV $^{\mathrm{a}}$ & Pvalue & PPa $^{\mathrm{a}}$ & Pvalue \\
\hline BMI & -.145 & .443 & -.283 & .173 \\
\hline Waist & -.087 & .152 & -.314 & .252 \\
\hline SBP & .753 & $<0.01$ & .853 & $<0.01$ \\
\hline DBP & .212 & .236 & -.005 & .976 \\
\hline Triglycerides & .231 & .257 & -.154 & .363 \\
\hline HDL-c & -.236 & .242 & -.245 & .144 \\
\hline FPG & -.065 & .758 & -.091 & .598 \\
\hline
\end{tabular}

a=Pearson's r, was used to analyze the correlation between AS variables and metabolic syndrome components.

On the other hand, we observed statistically significant correlations when we contrasted baPWV and PP with age $(\mathrm{r}=.378, \mathrm{p}=.043$ and $\mathrm{r}=.627, \mathrm{p}=<0.01$, respectively).

Among patients without MetS, no significant correlations were observed between baPWV and waist, triglycerides, HDL-c, FPG, SBP, or DBP. Only PP correlated with SBP and DBP (data not shown). 


\section{Discussion}

In the present study, we observed a strong association between AS (baPWV and PP) and SBP in patients with MetS. The association between AS and hypertension is evident, presumably as an adaptive response to an increased hemodynamic load (Gibbons, 1994). In contrast, another report (Quevedo, 2012) demonstrated that AS precedes the development of hypertension, although the mechanisms involved are not well established. Our findings are similar to those found in a recent study in which the component of MetS that most affected AS was hypertension [OR (95\%CI): 4.5 (4.0-5.1)] (Chen, 2015). Likewise, in a previous study that examined a population of 4985 patients with MetS, AS was consistently associated with three different clusters: high triglycerides, elevated blood pressure and abdominal obesity (TBW); low HDL-c, elevated blood pressure and abdominal obesity (HBW); and elevated fasting glucose, elevated blood pressure, and abdominal obesity (GBW) (Scuteri, 2014).In our study, we identified an important interaction between AS and elevated blood pressure independent of age; however, some have highlighted the importance of age in AS independent of blood pressure (Cho, 2015). Therefore, age and blood pressure, as well as their interaction, should be considered when evaluating AS.

The main limitation of this study is that we report analysis obtained solely from cross-sectional data. Thus, we cannot draw any conclusions about the role of the associations of blood pressure with baPWV and PP in patients with MetS as possible predictors of future clinical events. This is the first study conducted in a Mexican patient population that demonstrates that blood pressure is the component of MetS that is most strongly associated with AS.

\section{REFERENCES}

Allison MA, Budoff MJ, Wong ND, et al. (2008) Prevalence of and risk factors for subclinical cardiovascular disease in selected US Hispanic ethnic groups.Am J Epidemiol. 167. P. 962-969.

Barquera, S. Campos Nonato, I. Hernández-Barrera, et al. (2013) Prevalencia de obesidad en adultos mexicanos, ENSANUT 2012. Salud Pública Mex 55 Supl 2. P.S151-S160.

Chen L, Zhu W, Mai L, et al.(2015) The association of metabolic syndrome and its components with brachial-ankle pulse wave velocity in South China. Atherosclerosis. 240. P. 345-350.

Cho SK, Cho SK, Kim KH, et al. (2015) Effects of Age on Arterial Stiffness and Blood Pressure Variables in Patients with Newly Diagnosed Untreated Hypertension. Korean Circ J. 45. P. 44-50.

Gibbons GH, Dzau VJ. (1994) The emerging concept of vascular remodeling.N Engl J Med. 330. P. 1431-1438.

Laurent S, Boutouyrie P, Asmar R, et al. (2001) Aortic stiffness is an independent predictor of allcause and cardiovascular mortality in hypertensive patients. Hypertension. 37. 1236-1241.

Laurent S, Cockcroft J, Van Bortel L, et al. (2006) Expert consensus document on arterial stiffness: Methodological issues and clinical applications. Eur Heart J. 27. P. 2588-2605.

Quevedo KK. (2012) Aortic stiffness, blood pressure progression, and incident hypertension. Cardiol Rev.9. P. 875-81.

Scuteri A, Cunha PG, Rosei EA, et al. (2014) Arterial stiffness and influences of the metabolic syndrome: A cross-countries study. Atherosclerosis. 233. p. 654-660.

Tanaka H, Munakata M, Kawano Y, et al.(2009) Comparison between carotid-femoral and brachialankle pulse wave velocity as measures of arterial stiffness. J Hypertens.27.p 2022-2027.

Yamashina A, Tomiyama H, Takeda K, et al. (2002) Validity, reproducibility, and clinical significance of noninvasive brachial-ankle pulse wave velocity measurement. Hypertens Res. 25. 359-364. 